\title{
Mehrsprachigkeit in der Slowakei und die Stellung des Deutschen
}

\section{Katarína Fedáková}

The article deals first with the term "multilinguality" and then presents the results of two empirical studies concerning foreign language learning in the Slovak Republic and the role of foreign languages under Slovak circumstances. The main goal is to characterize the foreign language competence in the Slovak Republic generally and the influence of the mother tongue and other languages on learning of the target language.

Multilinguality - foreign language - foreign language teaching - foreign language learning foreign language competence - mother tongue

Der Artikel setzt sich zuerst mit dem Begriff der Mehrsprachigkeit auseinander und führt einige Ergebnisse zweier Umfragen in Bezug auf die Erforschung der Sprachkompetenz in der Slowakei sowie zum Einfluss der Muttersprache bzw. der zuvor gelernten Sprachen auf das Erlernen der Zielsprache.

Mehrsprachigkeit - Fremdsprache - Fremdsprachenunterricht - Fremdsprachenlernen Sprachkompetenz - Muttersprache

\section{Mehrsprachigkeit in der EU und der Slowakei}

Die Europäische Union (EU) setzt sich die Verwirklichung des Mehrsprachigkeitskonzepts als eines ihrer wichtigsten Ziele. Die erste Mitteilung der Kommission zum Thema „Eine neue Rahmenstrategie für Mehrsprachigkeit "1 wurde im November 2005 angenommen, wodurch der Aktionsplan der Kommission zur Förderung des Sprachenlernens und der Sprachenvielfalt (2004-2006) ergänzt wurde. In der Mitteilung (Language policy 2012) werden die drei Grundlinien der EU-Politik im Bereich der Mehrsprachigkeit formuliert:

1. den Bürgerinnen und Bürgern Zugang zu Rechtsprechung, Verfahren und Informationen der EU in ihrer Sprache gewähren,

2. die wichtige Rolle der Sprachen und der Mehrsprachigkeit in der europäischen Wirtschaft betonen und weiter stärken,

1 Die Definitionen dieses Begriffs sind uneinheitlich. Die EU bzw. der Europarat (ER) versuchen, die Unterscheidung zwischen multilingual und plurilingual in Bezug auf plurilinguale Individuen und multilinguale Gesellschaften durchzusetzen, was allerdings bis jetzt in der Spracherwerbsforschung sowie in der Sprachlehr- und Sprachlernforschung nicht angenommen wurde. Mißler (1999: 6) betrachtet Mehrsprachigkeit bzw. Plurilingualismus als übergeordnete Begriffe und Zweisprachigkeit/ Bilingualismus u. a bzw. Vielsprachigkeit/Multilingualismus als spezifische Ausprägungen von Mehrsprachigkeit/Plurilingualismus. Hier wird Mehrsprachigkeit im Sinne von Mißler aus der psycholinguistischen Perspektive verwendet, d. h als Erwerb der Mehrsprachigkeit und ihrer Auswirkungen auf das Lernverhalten eines Individuums und dessen kognitiven Fähigkeiten. 
3. alle Bürgerinnen und Bürger ermutigen, mehr Sprachen zu lernen und $\mathrm{zu}$ sprechen, um das gegenseitige Verstehen und die Kommunikation zu verbessern. ${ }^{1}$

Diese Forderungen lassen sich ziemlich schwer von oben verordnen. Sie können zwar von oben unterstützt, müssen allerdings von unten entwickelt werden. Krumm (Hufeisen/Neuner 2003: 37) als Kritiker dieses EU-Konzepts bezeichnet die Sprachenpolitik der EU als widersprüchlich: Die Diskussion über diese Politik werde seiner Meinung nach immer noch tabuisiert und man begnüge sich aus „,...] lauter Ratlosigkeit mit bloßer Mehrsprachigkeitsrhetorik“. Eine EurobarometerUmfrage zeigt, dass $47 \%$ der EU-Bürger keine Fremdsprache sprechen und zum Beispiel in Deutschland die Schüler im Durchschnitt nur 1,2 Sprachen pro Schüler lernen (Krumm, zit. nach Hufeisen/Neuner 2003: 36, 35).

In der Slowakei war die Situation nach dem EU-Beitritt im Jahre 2004 sehr günstig für eine bessere und realistischere Ausarbeitung und Realisierung des Mehrsprachigkeitskonzepts. Diese positiven Bedingungen ergeben sich aus der Geschichte des Landes, der geographischen Lage, der Zusammensetzung der Bevölkerung. Ihre Bedeutung sollte nach 2004 als eine „interkulturelle Notwendigkeit" (Profil 2009: 14) noch mehr hervorgehoben werden.

Allerdings verliefen, wie eine Expertenkommission der EU für die Slowakei in ihrem Bericht (Profil 2009: 15) feststellte, die Veränderungen im Jahre 1989 und nach 2004 in vielen Bereichen unter politischem Druck, was sich (wenn auch mit gewisser Verzögerung) vor allem im Bildungssystem zeigte. Viele politische Repräsentanten haben gleich nach der Wende die Forcierung des Englischen auf Kosten der Mehrsprachigkeit bevorzugt. Es überrascht also nicht, dass - wie Dolník (2004: 37) es ausdrückt - „[...] in der Slowakei dieses Thema [Mehrsprachigkeit] eher zufälligerweise angesprochen und [...] bislang lediglich als eine periphere Erscheinung behandelt wird." Im Bericht der Expertenkommission der EU für die Slowakei wurde die Beunruhigung darüber zum Ausdruck gebracht, dass die Zukunft der anderen Fremdsprachen außer Englisch in der Slowakei durch die offizielle Fremdsprachenpolitik bedroht ist (Profil 2009: 49).

Für die Stellung einer Fremdsprache spielen der Status/das Prestige sowie die Nützlichkeit eine wichtige Rolle. Dolník (2004: 41) definiert den Status als die Anzahl der Sphären, in denen die Sprache bevorzugt wird sowie den Grad ihrer Universalität. Am Beispiel des Deutschen soll dies verdeutlicht werden.

Anfang der 90er Jahre verlief z. B. nur 1\% der internen Kommunikation der EU auf Deutsch, obwohl Deutschland die wichtigste und stärkste Wirtschaft in der EU darstellt. Krumm (2003: 36) spricht von „einer schwer akzeptablen Hegemonie von Englisch und Französisch“. Die Stellung des Deutschen in der EU (und auch in der Welt) wird so bewertet, dass das Prestige dieser Sprache den realen

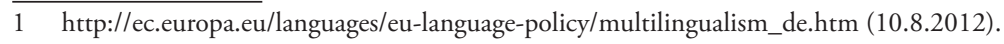


Existenzbedingungen nicht angemessen ist. Aber die Stellung einer Sprache wird, wie Muhr (2004: 58) anführt, auch von einem internen Faktor beeinflusst, der der jeweiligen Sprachgemeinschaft inhärent ist, nämlich von der Sprachloyalität: „Was nun die Sprachloyalität anbelangt, ist es gerade seit 1945 in Deutschland zu einer dramatischen Wende gekommen. Der beinahe fanatische Sprachstolz früherer Zeiten hat einer relativen Gleichgültigkeit Platz gemacht. Denn nach einer Umfrage, die Ende der 90er Jahre gemacht wurde, können sich 25 \% der Deutschen vorstellen, auch gänzlich auf ihre Sprache zu verzichten" (Muhr 2004: 58).

Im Gegensatz zu dieser Entwicklung in der Stellung des Deutschen scheint das Englische zu einer EU-Gemeinsprache, vielleicht sogar, wie es Dolník treffend auf den Punkt bringt, zu einem „Identifikationsmerkmal der europäischen Volkshaftigkeit“ zu werden (2004: 41). Die englische Sprache beeinflusst sehr stark das Konzept der Mehrsprachigkeit sowohl in der EU als auch in der Slowakei.

Der Sprachwechsel vom Deutschen zum Englischen setzte in der Slowakei gleich nach der Wende im Jahre 1989 ein. Die Zahl von Deutschlernenden an Grundund Mittelschulen sank in der ehemaligen Tschechoslowakei ständig, besonders zugunsten des Englischen, aber immer mehr auch zugunsten anderer Fremdsprachen wie Französisch, Spanisch und Russisch. ${ }^{1}$

2007 wurde von Seiten des slowakischen Schulministeriums eine neue Konzeption für den Fremdsprachenunterricht an Grund- und Mittelschulen (Reform 1 - R1, 2007) ausgearbeitet, um die Mehrsprachigkeit zu unterstützen. Ab 2008 sollten demnach die 1. Fremdsprache ab der 3., die 2. Fremdsprache ab der 5. Klasse unterrichtet werden. Zurzeit liegen einige Analysen für die erste Phase der R1 vor (vgl. Bockaničová et al. 2010: 6-7). ${ }^{2}$ Die ersten Analysen der Auswirkungen dieser ersten Phase der R1 vom Staatlichen pädagogischen Institut im Auftrag des Schulministeriums sind allerdings nicht sehr positiv. Da das Interesse an Englisch anstieg, wurde in diesen Analysen festgestellt, dass der Englischunterricht in der Primarstufe wegen des Mangels an qualifizierten Lehrern qualitativ schlecht und so für die Lernenden demotivierend ist. Er hat sogar einen negativen Einfluss auf den Muttersprachenunterricht bzw. auf das Erlernen einer zweiten Fremdsprache (vgl. Bockaničová et al. 2010: 6-7).

1 Im Jahre 2000 lernten insgesamt noch etwa 380000 Schüler Deutsch. Im Jahre 2005 verzeichnete man unter den Schülern einen Rückgang von mehr als $10 \%$ (340 000), wobei die Zahl der Deutschstudierenden etwa gleich blieb (12 500). Interessant war der Anstieg in der Kategorie der erwachsenen Lerner auf fast das Doppelte (von 6400 auf 12 000) (Ortner 2007: 43). Zu dieser Zeit war Englisch noch nicht obligatorisch die erste Fremdsprache.

2 Im Schuljahr 2008/2009 lernten in der dritten Klasse von 26553 Schülern 89,73 \% Englisch. Dieser Anteil sank in der 6. Klasse auf 69,07 \%. Im Schuljahr 2010/2011 stieg die Zahl der Englischlernenden in der dritten Klasse leicht auf $92,17 \%$ von 47610 Lernenden an, wobei in der 6. Klasse der Anteil der Englischlerner auf 64,02 \% sank. Deutsch wählten im Schuljahr 2008/2009 $9,89 \%$ in der dritten Klasse, in der sechsten Klasse waren es 26,28 \%.Im Schuljahr 2010/2011 konnte man für diese Fremdsprache in der dritten Klasse bereits eine Reduzierung auf 7,17 \% und in der 6. Klasse einen Anstieg auf 28,14\% verzeichnen. 
Im März 2011 trat die Novelle des Schulgesetzes (Reform 2 - R2) in Kraft und wird seit dem Schuljahr 2011/2012 in der Form realisiert, dass Englisch die einzige Pflichtfremdsprache in der Primarstufe ist, wobei nur die zweite Fremdsprache ab der 6. Klasse frei gewählt werden kann. An den Mittelschulen werden obligatorisch zwei Fremdsprachen gewählt. ${ }^{1}$

Wenn wir wollen, dass das Konzept der Mehrsprachigkeit eine Chance hat, kann nur eine effektive, zielgerichtete Sprachenpolitik aller betreffenden Institutionen und Medien, die das Lernen weiterer Fremdsprachen außer Englisch als attraktiv und als zu pflegende Alternative präsentieren, dabei behilflich sein. Wenn andere Fremdsprachen gelernt werden sollen, muss die zweite Fremdsprache, wie Lindemann (2007: 18) es formuliert, einen Status erhalten, „der sie davor schützt, als unwichtig und nebensächlich abgewertet werden zu können. “ Raasch (2002: 30) drückt dies treffend aus: „Mehrsprachig kann der Einzelne werden, aber ein Land benötigt die Vielsprachigkeit. Wenn die Einzelnen die Möglichkeit erhalten, je nach Interesse verschiedene Sprachen zu lernen, dann entsteht die Multilingualität des Landes aus der Plurilingualität seiner Bewohner. Wir brauchen Schulen, Hochschulen, Bildungsträger, die möglichst viele verschiedene Sprachen anbieten und in ihre Curricula aufnehmen."

Aus diesen Vorüberlegungen lassen sich mehrere Fragestellungen zu einigen Aspekten der Mehrsprachigkeit formulieren. Weiterhin werden einige davon fokussiert, und zwar: Wie ist der aktuelle Stand der fremdsprachlichen Kompetenz in der Slowakei? Wie beeinflusst die Muttersprache bzw. (eine) vorher gelernte Sprache(n) (meist handelt es sich um das Englische) das Lernen einer weiteren Sprache und wie ist die Stellung des Deutschen in der Slowakei (Argumente für bzw. gegen das Erlernen dieser Sprache)? Um Antworten auf diese Fragen zu finden, wurden einige Ergebnisse von zwei Umfragen ausgewählt und zu den o. g. Ausführungen in Beziehung gesetzt.

\section{Einige Aspekte der Mehrsprachigkeit - Darstellung und Interpretation der Untersuchungsergebnisse}

Ausgegangen wird von einigen ausgewählten Ergebnissen einer breit angelegten soziologischen Umfrage von Bútorová und Gyarfášová (2011, U1). Diese Umfrage hatte u. a. zum Ziel festzustellen, wie die von den Probanden deklarierte fremdsprachliche Kompetenz ist, welche positiven bzw. negativen Faktoren diese Kompetenz beeinflussen. Die Umfrage wurde im März 2011 vom Institut für öffentliche Fragen im Auftrag der Botschaft der Bundesrepublik Deutschland sowie

1 Da die R2 erst seit einem Jahr realisiert wird, liegen noch keine zuverlässigen Analysen der Auswirkungen vor. Nach meinen eigenen Erfahrungen aus persönlichen Kontakten mit Deutschlehrenden auf allen Stufen und Schultypen verschlechtert sich ihre Situation zunehmend. Die Anzahl der Lerngruppen sinkt drastisch und die Lehrkräfte werden nicht selten entlassen. 
des Goethe-Instituts in Bratislava unter 1086 Probanden aus der Slowakei ab dem 15. Lebensjahr (repräsentativ nach Alter, Geschlecht, Bildung, Nationalität, Größe des Wohnorts ausgewählt) durchgeführt. Da die Studie auf Slowakisch erschienen ist, wurden sämtliche hier angeführten Ergebnisse und deren Interpretation von mir übersetzt.

Die Resultate und Interpretationen aus der U1 sowie die in einigen Veröffentlichungen dargestellten Informationen werden durch einige im universitären Bereich ermittelte Angaben ergänzt bzw. mit ihnen verglichen. Umfrage 2 (U2, Fedáková 2010) wurde an der Philosophischen Fakultät der Universität Prešov im Wintersemester 2010 realisiert. Die Versuchsgruppe der U2 $(\mathrm{N}=100$, davon 79 weiblich, 21 männlich) bestand aus Deutschstudenten im ersten Semester, also aus erwachsenen, selbständigen Sprachenbenutzern auf der Stufe B1-B2 (vorgeschrieben durch das Abitur $)^{1}$ am Anfang ihres Hochschulstudiums. Alle Teilnehmer an der Untersuchung hatten ihre bisherige Sprachausbildung im Rahmen des slowakischen Bildungssystems absolviert. Die durchschnittliche Lerndauer von Deutsch betrug 7,68 Jahre, ${ }^{2}$ die außerschulischen Sprachkontakte mit den deutschsprachigen Ländern waren im Allgemeinen beschränkt.

Für die Datenerhebung in der U2 wurden einige ausgewählte Items aus drei Fragebögen benutzt, und zwar: Fragebogen zu Erfahrungen beim bisherigen Fremdsprachenlernen und beim Lernen einer neuen Fremdsprache (FEFL) (Mißler 1999: 339-343) und aus dem Fragebogen Beliefs About Language Learning Inventory (BALLI) (Horwitz 1999) und ein adaptierter Motivationsfragebogen, der auf Basis der gegenwärtigen Forschung von T. Schlak et al. (2002) entwickelt wurde. Die Untersuchungsergebnisse sind in diesem Fall nicht statistisch fundiert, deshalb sind die Interpretationen eher als Skizze der möglichen Tendenzen zu verstehen.

\subsection{Sprachkompetenz in der Slowakei ${ }^{3}$}

Ausgehend von der U1 (Bútorová und Gyarfášová 2011: 4-5) gaben die Probanden mit 7 ausgewählten Sprachen (Russisch, Deutsch, Französisch, Englisch, Spanisch, Italienisch) am häufigsten an, Russisch (54\%), dann Deutsch (49 \%)

1 Diese Charakteristik ist relativ. Die deklarierten Stufen entsprechen oft nicht der Realität, worüber sich die Hochschullehrer in ihrem Unterricht überzeugen können.

2 Auch diese Angabe ist mit Vorsicht zu genießen, weil im slowakischen Bildungssystem die Kontinuität der Fremdsprachenausbildung immer noch nicht gewährleistet ist. Auf diese Tatsache wird z. B. im Bericht der Expertenarbeitsgruppe der Europäischen Kommission für die Slowakei mit Nachdruck hingewiesen (vgl. Profil 2009:18-21).

3 „Die Statistiken zur Situation einer Sprache, zur Zahl der Sprecher und Zweit- und FremdsprachenlernerInnen sind immer problematisch. Ab wann beherrscht man eine Sprache so gut, dass man sie bei einer Befragung angibt? Aber sie sind eine Orientierung, geben zumindest die Relation wieder, wodurch einige Tendenzen deutlich werden“ (Krumm 2003: 37). In diesem Sinne sind auch diese Angaben zu verstehen. 
und erst an dritter Stelle Englisch (46\%) zu beherrschen. Das Kriterium für die Selbsteinschätzung waren die Schulnoten 1 bis $5 .{ }^{1}$

Tab. 1: U1 - Selbsteinschätzung der Sprachbeherrschungsstufe - Schulnoten, in \%

\begin{tabular}{|c|c|c|c|c|c|}
\hline & $\begin{array}{c}\mathbf{1} \\
\text { ohne Probleme } \\
\text { schriftlich und } \\
\text { mündlich }\end{array}$ & $\begin{array}{c}\mathbf{2} \\
\text { sehr gut, } \\
\text { fast ohne } \\
\text { Probleme }\end{array}$ & $\begin{array}{c}\mathbf{3} \\
\text { ganz } \\
\text { gut }\end{array}$ & $\begin{array}{c}\mathbf{4} \\
\text { schwach, aber ich kann } \\
\text { mich ein bisschen } \\
\text { verständigen }\end{array}$ & $\begin{array}{c}5 \\
\text { ich beherrsche } \\
\text { die Sprache } \\
\text { gar nicht }\end{array}$ \\
\hline R & 1 & 4 & 17 & 32 & 46 \\
\hline D & 2 & 5 & 15 & 27 & 51 \\
\hline E & 5 & 9 & 14 & 18 & 54 \\
\hline F & 1 & 1 & 1 & 4 & 93 \\
\hline I & 0 & 0 & 0 & 4 & 96 \\
\hline S & 0 & 0 & 1 & 2 & \\
\hline
\end{tabular}

Was das Sprachbeherschungsniveau betrifft (ausgehend von der Note 4 als der Mindestgrenze aufwärts) können sich $5 \%$ in vier bis sechs Sprachen verständigen, 16 \% können drei Sprachen, 32 \% beherrschen zwei Sprachen, 29 \% können sich nur in einer Sprache verständigen und schließlich beherrschen $19 \%$ keine von diesen Sprachen. Französisch, Italienisch und Spanisch sind nach dieser Umfrage die am wenigsten benutzten Fremdsprachen.

In der U2, in der alle Probanden Deutsch beherrschen, wurde auch der Frage nachgegangen, in welcher anderen Fremdsprache sich die Probanden noch verständigen können. Die Fremdsprachen wurden in dieser Umfrage nicht vorgegeben. Das Sprachbeherrschungsniveau bezieht sich auf die Schulnoten 3-4. Es wurden hier keine Noten 1 oder 2 angegeben.

Tab. 2: U2 - Beherrschung anderer Fremdsprachen außer Deutsch - in \% (einige Befragten beherrschen 2 oder 3 Fremdsprachen)

\begin{tabular}{|c|c|c|c|c|c|c|c|}
\hline $\begin{array}{c}\text { andere Fremdsprachen } \\
\text { als Deutsch }\end{array}$ & $\begin{array}{c}\text { keine } \\
\text { andere FS }\end{array}$ & Engl. & Franz. & Russ. & Ungar. & Span. & Lat. \\
\hline & 5 & 90 & 7 & 5 & 2 & 2 & 2 \\
\hline
\end{tabular}

Hier hat Englisch eine eindeutig dominante Position, was den Schlussfolgerungen in der U1 entspricht, dass Englisch jüngere und höher gebildete Lerner auswählen

1 Es wird dabei davon ausgegangen, dass die meisten Lernenden ihre Kompetenz realistisch einschätzen können. Es handelt sich hier um keine objektiv existierende Kompetenz, sondern um eine subjektive Wahrnehmung davon (Mißler 1999: 179-180). 
(Bútorová und Gyarfášová 2011: 5). Französisch, Italienisch und Spanisch sind nach der U1 die am wenigsten angegebenen Fremdsprachen. Die Lehrenden an den Mittelschulen berichten aber bei der Wahl einer zweiten Fremdsprache seitens der Lernenden von einem großen Anstieg des Interesses besonders an Spanisch auf Kosten des Deutschen. In der U2 nahm Französisch ziemlich überraschend die zweite Stellung ein, meistens als die dritte Fremdsprache angegeben. Diese Sprache hatte in der Slowakei bisher keine nennenswerte Tradition, was sich in den letzten Jahren zumindest in der Westslowakei durch die Wirkung französischer Automobilhersteller in Trnava ändert.

\subsection{Der Einfluss der Muttersprache bzw. der vorher gelernten Sprachen}

In den letzten zwanzig Jahren hat man die Möglichkeiten erkannt, die sich aus dem Miteinander der Sprachen ergeben (vgl. z. B. Hufeisen 1999, Macaire 1999, Lutjeharms 1999). Es wird eingesehen, dass durch die Lerngeschichte wichtige Möglichkeiten in Bezug auf die nächsten Sprachen eröffnet werden.

\subsubsection{Der Einfluss der Muttersprache}

Diese Variable wurde in der U2 untersucht. Die Auswirkung des Slowakischen, das selbst zu den schwierigen Sprachen zählt, wird mit Hilfe von folgenden Aussagen und einer 5-stufigen Likert-Skala (von starker Ablehnung bis zur starken Zustimmung) ermittelt: Die Muttersprache stört beim Lernen einer weiteren Sprache. Mit dieser Aussage erklärten sich $36 \%$ einverstanden, $21 \%$ konnten sich nicht entscheiden und $43 \%$ lehnten sie stark bis sehr stark ab. Hier fällt auf, dass der Einfluss der Muttersprache weder als eindeutig hilfreich noch als eindeutig störend eingeschätzt wird.

Die veröffentlichten Untersuchungsergebnisse und ihre Interpretationen diesbezüglich sind unterschiedlich. Nach einigen Studien (in Hufeisen 2004: 22) nimmt die Muttersprache anscheinend eine andere Stellung im mentalen Lexikon und in der Sprachverarbeitung ein als Fremdsprachen. Man schaltet beim Lernen einer Fremdsprache offenbar auf den sog. „foreign language mode“ um. Diese Annahme wird von der Neurologie untermauert: Die später gelernten Fremdsprachen werden gemeinsam in einem anderen Teil des Gehirns verarbeitet als die Muttersprache und die früh gelernten Fremdsprachen. Auf der anderen Seite ist Hufeisen (2004: 22-23) überzeugt, dass man die Muttersprache im Kopf der Lernenden nicht abschalten kann. Wildenauer-Józsa (2004: 38) führt die Ergebnisse ihrer Interviewstudie mit erwachsenen Deutschlernenden an, in der diese zum Thema Sprachvergleich befragt wurden. Im Bereich der Grammatik waren die Probanden in der Lage, 73,5 \% Ähnlichkeiten und 64,7 \% Unterschiede zwischen deutscher und muttersprachlicher Grammatik, unabhängig von ihrer Muttersprache, zu nennen. Hier könnte eine 
wichtige Rolle die Tatsache spielen, dass es sich um erwachsene Lernende handelt, die sich das System ihrer Mutersprache bereits angeeignet haben und so besser den Sprachvergleich nutzten können.

Nichtsdestotrotz sollte die Aufgabe der slowakischen Fremdsprachenlehrenden sein, in ihrem Unterricht nach Möglichkeiten eines kontrastiven oder vergleichenden Vorgehens in Bezug auf die Mutter- und die Zielsprache zu suchen, um möglichst viele Zugänge zu dieser Zielsprache im Sinne der Lernökonomisierung sowie effektivierung zu nutzen (insbesondere bei dem Aussprachetraining).

\subsubsection{Der Einfluss der vorher gelernten Sprache(n)}

In der U2 wurde der Einfluss der vorher gelernten Sprachen mit der Aussage ermittelt: Es ist leichter, eine neue Fremdsprache zu erlernen, wenn man schon eine andere Fremdsprache beherrscht. Die meisten Probanden (61 \%) sind der Auffassung, dass ihnen die zuvor gelernten Sprachen bei der Aneignung einer neuen Sprache weiterhelfen. Nicht entscheiden konnten sich $28 \%$ und $11 \%$ lehnten diese Aussage ab. Die Probanden sind also eher vom positiven Einfluss der zuvor gelernten Sprachen als der Muttersprache überzeugt. Diese Angaben sollte man allerdings mit Vorsicht interpretieren, weil es sich nicht um verifizierte Nachweise handelt, sondern um deklarierte Einstellungen dazu.

Das Englische ist, wie erwähnt, eine starke (Schul)Fremdsprache. In der U1 wird sie zwar an die dritte Stelle hinter Russisch und Deutsch gestellt, was ihre Beherrschung in der Versuchsgruppe betrifft, sind allerdings fast alle Befragten (95\%) überzeugt, dass Englisch wichtig bis eher wichtig ist (Bútorová und Gyarfášová 2011: 10). Deswegen sollte weiterhin der Einfluss des Englischen auf das Lernen einer weiteren Fremdsprache (in der U2 vor allem des Deutschen) hinterfragt werden. In der U2 schätzt fast die Hälfte (44\%) der Befragten vorhandene Englischkenntnisse als hilfreich oder eher hilfreich ein. Als weder hilfreich noch störend wird das Englische von $23 \%$ angesehen, als störend bis sehr störend von $10 \%$ und $15 \%$ äußerten sich nicht dazu. Interessant ist, dass die Einstellungen zum Einfluss des Englischen auf das Erlernen weiterer Fremdsprachen wider Erwarten nicht eindeutig positiv waren. Man hätte hier erwartet, dass sich einige in der Fachliteratur angeführte Erkenntnisse bestätigen: „Ein zwischensprachlicher Einfluss wurde am häufigsten unter der Voraussetzung festgestellt, dass keine Ähnlichkeiten ${ }^{1}$ zwischen der Muttersprache und der ersten Fremdsprache sowie zwischen der Muttersprache und der zweiten Fremdsprache existieren, wohingegen die erste und zweite Fremdsprache erhebliche Ähnlichkeiten aufweisen“ (Mißler 1999: 29). Dies sollte eigentlich für die Kombination Englisch und Deutsch als Fremdsprachen und Slowakisch als

1 Ähnlichkeit wir hier nicht im Sinne von „tatsächlich existierende Ähnlichkeit“, sondern als „vom Lernenden subjektiv wahrgenommene Ähnlichkeit“ verstanden. 
Muttersprache sprechen. ${ }^{1}$ Auf der anderen Seite fühlen sich die Lernenden gerade bei solchen als ähnlich wahrgenommenen Fremdsprachen oft von Verwechslungen oder Verwirrungen wegen (zu) großer Ähnlichkeit gestört. Dieser Umstand könnte auch die nicht eindeutige Einstellung zum Einfluss des Englischen in der U2 erklären.

Man sollte sich für den Unterricht überlegen, wann die gelernte(n) Fremdsprache(n) als Hilfe, wann zum Kontrast oder als Verbindung zu der Zielsprache eingesetzt werden können, bzw. wann die Fremdsprache(n) besser in den Hintergrund gestellt oder sogar besser ausgeschlossen sein soll(en). In Bezug auf die behandelte Problematik der Mehrsprachigkeit könnte man einige weiterführende Fragestellungen verfolgen. Es wäre z. B. interessant zu untersuchen, welches Vorwissen und welche Vorerfahrungen unter welchen Bedingungen als positiv bzw. negativ empfunden werden. In diesem Zusammenhang sollte man zwischen den sprachbezogenen Vorerfahrungen und Vorkenntnissen sowie den fremdsprachenlernspezifischen Erfahrungen (Lernstrategien, Bewusstsein u. a.) unterscheiden (vgl. Hufeisen 2004: 20). Krumm (1995: 198, zit. in Hufeisen 1999: 4) drückt dies folgendermaßen aus: „Ob und in welchem Ausmaß bei früheren Sprachen gelernte Strategien und Fertigkeiten tatsächlich problemlos auf neue Sprachen transferierbar sind, ist noch keineswegs geklärt. Sicher ist jedoch, dass solche Sprachlernerfahrungen die Erwartungen an den Unterricht in einer neuen Sprache nachhaltig prägen."

Weiter ließe sich untersuchen, wie subjektiv die Wahrnehmung der Ähnlichkeit zwischen der Mutter- und der Zielsprache oder zwischen der ersten und der zweiten Fremdsprache ist; welche Rolle die Dominanz einer Fremdsprache, das erreichte Kompetenzniveau in der Zielsprache u. a. spielen (vgl. Mißler 1999: 15-109). Die Praxis zeigt, wie wenig noch die Zusammenhänge zwischen konkreten Sprachen erforscht sind und wie weit wir noch von einer "praktikablen Mehrsprachigkeit“ (Keilholz-Rühle 1999: 52) entfernt sind.

\subsection{Argumente für bzw. gegen das Erlernen der deutschen Sprache}

Wie schon erwähnt, sinkt das Interesse an der deutschen Sprache kontinuierlich. Was bewegt die Lerner dazu, dass sie sich trotzdem für Deutsch entscheiden? Ausschlaggebend dafür sind in der U1 das Alter und die Ausbildung. Eher ältere Probanden (über 45 Jahre) mit einer niedrigeren Ausbildung beherrschen Deutsch besser als Englisch. Bei den jüngeren und höher qualifizierten Befragten dominiert dagegen eindeutig Englisch (Bútorová und Gyarfášová 2011: 11). Im Folgenden werden die Gründe gegen bzw. für das Erlernen des Deutschen näher beschrieben.

1 Nach Huneke und Steinig (2007: 219) wird Englisch als besonders hilfreich im Wortschatzbereich bezeichnet. 


\subsubsection{Argumente gegen das Erlernen der deutschen Sprache}

Als Kontra-Argumente geben die meisten befragten Personen in der U1 (Bútorová und Gyarfášová 2011: 8), die Deutsch nicht beherrschen, an, keine Möglichkeit zum Deutschlernen gehabt zu haben $(41 \%) .{ }^{1}$ Fünfzehn Prozent sind überzeugt, dass sie für Sprachen nicht begabt sind (hierbei handelt es sich vor allem um Probanden mit niedrigerer Bildung). Die gleiche Prozentzahl verlernte mit der Zeit die in der Kindheit erworbene Kompetenz. Für $11 \%$ hat Deutsch in ihrem Leben keine Bedeutung. Vor allem jüngere Probanden finden Deutsch nicht schön (6 \%) und glauben, Deutsch sei eine schwere Sprache (6\%). ${ }^{2}$

In der U2 sind $98 \%$ der Befragten der Meinung, dass Deutsch sehr schwer bis mittelschwer ist. Nur $2 \%$ glauben, dass diese Sprache leicht ist. Es gibt in der Stichprobe keinen Probanden, der Deutsch für eine sehr leichte Sprache hält.

Wie aus diesen Angaben hervorgeht, genießt Deutsch - und dies nicht nur in der Slowakei - den Ruf, „[...] eine sprachlich wie kulturell schwer zugängliche Sprache zu sein" (Krumm 2003: 37). Diese klischeehafte Wahrnehmung wird meistens vor dem Beginn des Lernens ausgeprägt. Es stimmt, dass das Deutschlernen ein kontinuierliches und intensives Arbeiten erfordert, noch mehr als das Englische (Feuerhake et al. 2004). Mit der Zeit sehen deshalb einige Schüler ein, dass sie keine schnellen, beobachtbaren Lernerfolge erreichen sowie keine praktischen Anwendungsmöglichkeiten für den Einsatz der deutschen Sprache innerhalb und außerhalb des Unterrichts sehen, was zum Sinken der persönlichen Motivation bzw. sogar zum Abbruch des Lernens führen kann. ${ }^{3}$

Dem widersprechen scheinbar die Ergebnisse der U1, in der 88 \% der Probanden der Ansicht sind, dass es sehr bis eher wichtig ist, auch Deutsch zu beherrschen (Englisch ist zweifelsohne die wichtigste Fremdsprache mit $95 \%$, Russisch belegt mit 39 \% den dritten Platz) (Bútorová und Gyarfášová 2011: 11). Das ist vielleicht damit zu erklären, dass es eine Diskrepanz zwischen rationalen Argumenten und dem persönlichen Willen oder der Lust, Deutsch tatsächlich zu lernen, gibt. Oder spielt

1 An den von den Befragten besuchten Schulen wurde kein Deutsch unterrichtet. Wie in der Analyse der Auswirkungen der Fremdsprachenreform in den Grundschulen (Bockaničová/De Jaegher/ Duranová et al. 2010: 6) festgestellt wird: „[...] die Druckpolitik der entscheidenden Instanzen sowie die Unmöglichkeit, einen qualifizierten Lehrer zu sichern, zwingt die Schuldirektoren, auf der Primarstufe ein begrenztes Repertoire an Fremdsprachen anzubieten "(Übersetzung K. Fedáková).

2 Ortner (2006: 51) relativiert die Einstellung zum Deutschen als einer schweren Sprache mit einem Gegenargument aus Claudia Riemers Russlandstudie: „Gemessen an Russisch ist Deutsch nicht schwer, ganz im Gegenteil ... Deutsch ist ebenso leicht wie jede andere Sprache, vorausgesetzt, der Unterricht hält sich an ein paar wenige gesicherte Grundlagen und an die Interessen der Schüler - schnelle Ergebnisse sichern, täglich die kleinen Erfolge sichtbar machen, das Lernen lernen und damit auch den Lernerfolg sichern."

3 Vom Englischen ist man überzeugt, dass man es, nebst allen anderen Vorteilen, relativ schnell erlernen kann. Eine andere Frage ist allerdings, ob sich die Englischlernenden dessen bewusst sind, dass man zwar für Englisch als lingua franca vier bis fünf Jahre, für Englisch als lingua culturalis allerdings acht bis zehn Jahre braucht (Raasch 2002: 31). 
hier die soziale Erwünschtheit, auf bestimmte Fragen positiv zu antworten, eine Rolle? (Es handelte sich ja um eine Umfrage mit dem Schwerpunkt auf Deutsch.) Man kann lediglich hoffen, dass es in ein paar Jahren auch in der Slowakei zu einer ähnlichen Situation kommt wie in einigen anderen Ländern, wo das Lernen von Deutsch heutzutage wegen seines „exotischen“ Charakters mehr geschätzt wird als das Englische, das ,jeder kann“, wie z. B. in Schweden: „[...] es klingt besser, wenn man sagt, dass man Deutsch lernt, als wenn man Englisch lernt [...]” (Kirchner 2004: 18). Ortner (2007: 44) benutzt einen Ausdruck aus Riemers Länderstudie und nennt die entsprechende Motivation „instrumentelles Exotenmotiv“.

\subsubsection{Argumente für das Erlernen der deutschen Sprache}

Da Deutsch als eine (sehr) schwierige Sprache wahrgenommen wird, ist die Motivation für das Erlernen dieser Sprache von großer Bedeutung. Viele Lerner entwickeln erst kurz vor oder nach der Beendigung ihrer Schulzeit selbstbestimmte Formen der Motivation, wobei Berufsmöglichkeiten und Auslandsaufenthalte eine wichtige Rolle spielen (vgl. Riemer 1997: 9). Eine Rolle für die Lernmotivation eines Fremdsprachenlernenden stellt die Qualität und die Häufigkeit seiner Kontakte mit den Muttersprachlern bzw. mit der Zielsprachenkultur dar.

Von den angegebenen Pro-Argumenten für Deutsch wählten die Probanden in der U1 (Bútorová und Gyarfášová 2011: 6-8) auf den Plätzen 5 und 6 die Aussagen, dass Auslandsaufenthalte sowie die positive Einstellung zum Zielspracheland und seiner Kultur eine Rolle bei ihrer Wahl spielten.

In der U2 waren die persönlichen Kontakte der Probanden mit den deutschsprachigen Muttersprachlern recht eingeschränkt, weshalb sie in einer anderen Form untersucht wurden. Über 60 \% der Befragten sehen sich häufig bzw. sehr häufig deutsche Sendungen im Fernsehen und/oder deutsche Filme an. Mehr als ein Drittel gibt überraschend an, häufig deutsche Zeitungen oder Zeitschriften zu lesen. Bücher werden gelegentlich von knapp einem Drittel der Probanden gelesen Ähnliches gilt für die Musik. An letzter Stelle ist Radio, das von mehr als einem Drittel der Befragten nie gehört wird.

Das Argument an 4. Stelle in der U1 bezieht sich auf die Möglichkeit, relativ früh mit dem Deutschlernen zu beginnen. Mehr als ein Drittel (38 \%) hält das Erlernen einer Fremdsprache in den ersten drei Klassen der Grundschule für optimal. Fast der gleiche Prozentanteil bezieht sich auf das Vorschulalter. ${ }^{1}$ Allerdings wird hier nicht ermittelt, welches Alter am besten für den Erwerb einer zweiten Fremdsprache ist. Es herrscht zwar die Meinung vor, dass die parallele Entwicklung von zwei Sprachen die Kinder zu sehr belastet oder zu kognitiven Defiziten führen

1 Das Vorschulalter wird nach der U1 (Bútorová und Gyarfášová 2011: 10) von 33 \% Männern (mit Hochschulbildung $47 \%$ ) und $41 \%$ Frauen (mit Hochschulbildung sogar $58 \%$ ) für das beste Alter für das Fremdsprachenlernen gehalten. 
kann, das konnte aber von der Forschung eindeutig widerlegt werden (Steinig und Huneke 2007: 222). Die Möglichkeit des frühen Fremdsprachenunterrichts verlangt gut ausgebildete Lehrer, Zeit für den neuen Stoff und Kontinuität in den Schulen. Die Kinder können dadurch früh mit mehreren Sprachen konfrontiert und für die Mehrsprachigkeit sensibilisiert werden (zu dieser Zeit natürlich noch ohne systematischen Spracherwerb). In der U2 wurde dieser Aspekt explizit nicht ermittelt. Aber aufgrund der durchschnittlichen Lerndauer kann man darauf schließen, dass die Befragten relativ früh mit dem Deutschlernen anfingen, allerdings nur in einigen wenigen Fällen im Vorschulalter.

An dritter Stelle in der U1 ist überraschend die Begründung, dass die Probanden Deutsch lange genug und kontinuierlich lernen konnten. Dies steht in einem gewissen Widerspruch zum Bericht der Expertenkommission des ER für die Slowakei (vgl. 2009: 20), wonach in den Jahren 2003-2004 80 \% und mehr der Englisch- und Deutschlernenden zwar die Möglichkeit hatten, ihr Fremdsprachenlernen auch auf der Mittelschule fortzusetzen, aber mehr als ein Drittel von ihnen wieder von Null anfing. Es wäre interessant, die Gründe dafür zu ermitteln.

Das zweitwichtigste Argument unter den Pro-Argumenten in der U1 (Bútorová und Gyarfášová 2011: 8) sind die positiven Lernerfahrungen und das hohe Niveau des Deutschunterrichts in den slowakischen Schulen, was zu der wichtigen intrinsischen Motivation ${ }^{1}$ führen kann. Auch Weinberger (2007: 540) betont, dass positive schulische Erfahrungen und der frühe Beginn des Deutschunterrichts die Motivation zum Lernen unterstützen. Diese Argumente bestätigt ebenfalls Lindemann (2007: 18) in ihrer Studie, dass für die Aufrechterhaltung und ggfs. die Stärkung der Motivation und der Lernbereitschaft die fachliche, sprachliche und didaktische Kompetenz des Lehrers eine wesentliche Rolle spielen.

Das wichtigste Argument für das Erlernen der deutschen Sprache in der U1 bezieht sich auf die Notwendigkeit, die deutsche Sprache aus beruflichen Gründen zu beherrschen, also die Probanden sind instrumentell motiviert. ${ }^{2}$

Diese Ergebnisse wurden im Grunde genommen auch in der U2 bestätigt. Hier nimmt die instrumentelle Motivation den zweiten Platz ein. Die höchste durchschnittliche Zustimmung erreicht der Aspekt der intrinsischen Motivation. Es könnte angenommen werden, dass externer Leistungsdruck die intrinsische Motivation negativ beeinflussen kann (Schlak et al. 2002: 14). Einige Forschungsergebnisse (vgl. Kirchner 2004: 20) beweisen aber, dass so motivierte Lerner weniger Probleme haben, sich mit Lernmisserfolgen auseinanderzusetzen. Sie beziehen sie nämlich nicht so oft auf ihre mangelnde Kompetenz, sondern auf

1 Intrinsische Motivation bedeutet, das Lernen bereitet den Lernern Spaß, weckt ihre Neugier, ist interessant und motivierend. Sie halten den Fremdsprachenunterricht und seine Inhalte für relevant als Lernmotiv.

2 Instrumentelle Motivation wird als das Bedürfnis der Lerner verstanden, eine Fremdsprache aus pragmatischen Gründen zu lernen. 
äußere Umstände. Eine Kombination der intrinsischen Motivation mit externen Anregungen wird deswegen für sehr stabil gehalten.

\subsubsection{Bedeutung des Deutschen als erster bzw. als zweiter Fremdsprache im Vergleich zu anderen Fremdsprachen}

In Bezug auf die Frage Wenn alle Bewohner der Slowakei zwei Weltsprachen beherrschen sollten, welche Sprachen würden Sie für ihre Kinder auswählen (U1, Bútorová und Gyarfášová 2011: 15), würden sich $76 \%$ der Probanden für Englisch als erste Fremdsprache entscheiden. Nur 19 \% geben an, die Schulkinder sollten Deutsch als erste Fremdsprache lernen, und zwar mit folgenden Argumenten: „Deutsch ist wichtig für den Beruf und das Leben“ - $57 \%$; „wenn man die Grundlagen des Deutschen beherrscht, kann man leichter Englisch lernen“ - $15 \%$ und „es ist besser, zuerst eine schwierigere Sprache zu lernen und danach mit einer leichteren weiterzumachen" - $15 \%$ ". Für Deutsch als erste Fremdsprache sprechen auch die Ergebnisse mehrerer Forschungsprojekte (z. B. Rost-Roth 2003:126 und Macaire 1999: 9), die zeigen, dass die Lerner mit Deutsch als erster Fremdsprache bei einem Leistungsvergleich signifikant bessere Leistungen erreicht haben, weil verschiedene Lernstrategien effektiver aktiviert werden und das Sprachbewusstsein erhöht wird.

Wenn man das steigende Interesse an anderen Fremdsprachen (Russisch, Spanisch ggfs. Französisch) bedenkt, würden sich in der Slowakei zurzeit immerhin 57 \% für Deutsch als zweite Fremdsprache entscheiden (U1, Bútorová und Gyarfášová 2011: 15). Es entspricht dem weltweiten Trend, dass sich etwa drei Viertel aller Lernenden das Deutsche erst als zweite Fremdsprache (Tönshoff 2000: 57) aneignen. Fast 90 $\%$ der Probanden denken, dass es wichtig ist, beide Sprachen zu sprechen. Zwischen der Beherrschung von Englisch und Deutsch wurde in der U1 (S. 5) ein statistisch hoch signifikantes Verhältnis festgestellt. Allerdings nur $4 \%$ können diese Sprachen ohne oder fast ohne Probleme benutzen; 32 \% verfügen über elementare Kenntnisse in beiden Sprachen; 38 \% beherrschen weder Deutsch noch Englisch.

\section{Abschließende Bemerkungen}

Krumm (2003: 37) ist überzeugt, dass das Deutsche unter Berücksichtigung verschiedener Aspekte eine „starke (europäische Regional-)Sprache“ ist: Nach der Zahl der Muttersprachensprecher liegt Deutsch unter ca. 6000 Sprachen der Welt auf Platz 11 oder 12, in Bezug auf den wirtschaftlichen Einfluss sogar auf Platz 6 oder 7. Für die Slowakei ist das deutschsprachige Ausland wirtschaftlich besonders interessant, weil etwa $25 \%$ des slowakischen Exports in diese Länder gehen. Deutsch (mit 100 Millionen Muttersprachlern) ist weiterhin nach Englisch die meistbenutzte Sprache im Internet (Ortner 2007: 45). Auch in der U1 (Bútorová und Gyarfášová 2011: 11) wurde festgestellt, dass die häufigen Internetbenutzer 
mehr von der Notwendigkeit der Beherrschung des Deutschen überzeugt sind als diejenigen, die Internet weniger oder gar nicht benutzen. ${ }^{1}$

Deutsch als zweite Fremdsprache in einem Mehrsprachigkeitskonzept kann nur von angemessen ausgebildeten Lehrkräften vermittelt werden. Für den zeitgemäßen Mehrsprachigkeitsunterricht sollten, wie Krumm (2003: 46) es formuliert, „[...] die Konzepte der Lehrerausbildung so entwickelt werden, dass in Zukunft Lehrende als Experten in Sachen Mehrsprachigkeit auch bei wechselnden Sprachkonstellationen in der Lage sind, Sprachenvielfalt zu fördern“. Sind aber die germanistischen Institute $^{2}$ in der Slowakei in der Lage, diese neuen Aufgaben im Einklang mit den veränderten Anforderungen von Leben und Beruf zu berücksichtigen? Wie kann man angehende Deutschlehrer auf diese Unterrichtsrealität vorbereiten? Und schließlich, wie reflektieren die zugänglichen Unterrichtsmaterialien diese aktuellen Entwicklungstendenzen?

\section{Literaturverzeichnis}

Bockaničová, Katarína / De Jaegher, Darina / Duuranová, Denisa / Duuriš, Boris / Hoangová, Sabina / Hric, Martin / Vaškaninová, Katarína (2010): Analýza dopadu zavedenia povinnej výučby cudzieho jazyka v 3. a 6. ročníku základných škôl. Kvalifikovanost učitelov a dopad na kvalitu vzdelavania v oblasti cudzich jazykov, 19. 10. 2010. Bratislava, Štátny pedagogický ústav. www.educj.sk/buxus/.../Analyza_dopadov_zavedenia_CJ_v_ZS.pdf (20.7. 2012).

Brenner, Karsten (2001): Der europäische Hochschulraum als Chance und Herausforderung. In: Entwicklung von Curricula mit Auslandsbezug. Beiträge einer Fachtagung in Würzburg am 10. und 11. September 2001. Bonn, Deutscher Akademischer Austauschdienst. S. 14-25. Bútorová, Zora / Gyarfášová, Olga (2011): Jazyková kompetentnost na Slovensku: Nemčina $v$ porovnani s inými jazykmi. Bratislava, Goethe-Institut. [U1]

Dolník, Juraj (2004): Sprachenpolitik der EU, interkulturelle Germanistik Herausforderung für die slowakische Germanistik. In: Zbornik prispevkov zo VII. konferencie Spoločnosti učitelov nemeckého jazyka a germanistov Slovenska, Banská Bystrica, 1.- 4. 9. 2004. Banská Bystrica, Spoločnosṫ učitelov nemeckého jazyka a germanistov Slovenska. S. 37-45. Fedáková, Katarína (2010): Motivation für Deutsch als zweite Fremdsprachen. Ergebnisse einer Hochschulumfrage. Prešov: Prešovská univerzita, Ms. [U2].

Hallet, Wofgang / Königs, Frank, G. (Hg.) (2010): Handbuch Fremdsprachendidaktik. SeelzeVelber, Kallmeyer/Klett.

Horwitz, Elaine (1987): Surveying Student beliefs About Language Learning. In: Wenden, Anita / Rubin, Joan (eds.): Learner Strategies in Language Learning. Englewood Cliffs, NJ, Prentice Hall. S. 119-129.

1 Deutsch ist allerdings nach 1920 stark als Sprache der Wissenschaft und als Sprache von geistigen Produkten zurückgegangen (insbesondere in Technik und Naturwissenschaften, weniger in Medizin sowie in Geistes- und Sozialwissenschaften) (Muhr 2004: 58).

2 In der Slowakei gibt es acht germanistische Institute (die meisten in der Westslowakei: Bratislava, Trnava, Nitra, Komárno, in der Mittelslowakei in Banská Bystrica und Ružomberok, in der Ostslowakei in Košice und Prešov). Alle bieten auch die Deutschlehrerausbildung an. Vor dem Hintergrund der erwarteten Rückgänge an Studierenden dieser Studienrichtung sind es eindeutig zu viele. 
Horwitz, Elaine (1988): The beliefs about language learning of beginning university foreign students. In: Modern Language Journal, 72/3. S. 283-294. Auch unter: Wiley-Blackwell http://onlinelibrary.wiley.com.

Hufeisen, Britta / Neuner, Gerhard (2003): Mehrsprachigkeitskonzept - Tertiärsprachen Deutsch nach Englisch. Strassburg, Europarat.

Hufeisen, Britta (2004): Deutsch und die anderen (Fremd)Sprachen im Kopf der Lernenden. Wie man dieses Potenzial nutzen kann. In: Fremdsprache Deutsch. Zeitschrift für die Praxis des Deutschunterrichts 31/2004. S 19-23.

Huneke Wolfgang / Steinig, Hans-Werner (2007): Sprachdidaktik Deutsch. Eine Einführung. 3. Aufl. Berlin, Erich Schmidt Verlag.

Janíková, Věra (2011): Výuka cizích jazykư. Praha, Grada.

Klúčové údaje o vyučovaní jazykov v európskych školách. Edícia 2008 (2008). Brüssel, Education, Audiovisual and Culture Executive Agency.

http://eacea.ec.europa.eu/education/eurydice/documents/key_data_series/095SK.pdf (11.8 2012).

Krumm, Hans-Jürgen (2003): Sprachenpolitik und Mehrsprachigkeit. In: Mehrsprachigkeitskonzept-Tertiärsprachen - Deutsch nach Englisch. Strassburg, Europarat. S. 35-50.

Krumm, Hans-Jürgen: Interview mit Hans-Jürgen Krumm. http//www.goethe.de/ges/spa/.../ de4568512.htm (21. 1. 2011).

Language policy: http://ec.europa.eu/languages/eu-language-policy/multilingualism_de.htm (10. 8. 2012).

Le Pape Racine, Christine (2003): Mehrsprachigkeit und Immersion. Versuch einer Systematisierung lerntheoretischer und didaktischer Grundlagen sowie äußere Rahmenbedingungen auch aus schweizerischer Sicht. In: Hufeisen, Britta / Neuner, Gerhard (Hg.): Mehrsprachigkeitskonzept - Tertiärsprachen - Deutsch nach Englisch. Strassburg, Europarat. S. 105-130.

Lindemann, Beate (2007): Motiviert für Deutsch? Eine qualitative Studie zum Anfängerunterricht Deutsch als Fremdsprache in Norwegen. In: Zeitschrift für Interkulturellen Fremdsprachenunterricht 12/1/ 2007. http://zif.spz.tu-darmstadt.de/jg12-2/navigation/startbei.htm.

Lutjeharms, Madeline (1999) Tertiärsprache und Sprachbewusstheit. Was Lernende über den Einfluss der ersten Fremdsprache denken. In: Fremdsprache Deutsch. Zeitschrift für die Praxis des Deutschunterrichts 1/1999. München, Goethe- Institut/Stuttgart, Klett Verlag. S. 7-11.

Macaire, Dominique (1999): Erziehung der Kinder zur Mehrsprachigkeit: Wahn oder Sinn? In: Fremdsprache Deutsch. Zeitschrift für die Praxis des Deutschunterrichts 1/1999. München, Goethe- Institut / Stuttgart, Klett Verlag. S. 48-50.

Merkelbach, Chris (2011): Wie unterscheiden sich die Lernstrategien beim Erlernen von L2 und L3? Ergebnisse einer empirischen Studie bei taiwanischen Deutsch-als-L3Lernenden. In: Zeitschrift für Interkulturellen Fremdsprachenunterricht 16/2. S. 126-146. http://zif.spz.tu-darmstadt.de/jg-16-2/beitrag/merkelbach.pdf. (20. 7. 2012)

Mißler, Bettina (1999): Fremdsprachenlernerfahrungen und Lernstrategien. Eine empirische Untersuchung. Tübingen, Stauffenburg Verlag / Brigitte Narr.

Muhr, Rudolf (2004): Das Deutsche im Kontext der europäischen Sprachpolitik zwischen Konkurrenz und Kooperation. In: Zbornik prispevkov zo VII. konferencie Spoločnosti učitelov nemeckého jazyka a germanistov Slovenska. Banská Bystrica, Spoločnosť učitelov nemeckého jazyka a germanistov Slovenska. S. 56-65. 
Neuner, Gerhard (2003): Mehrsprachigkeitskonzept und Tertiärsprachendidaktik. In: Hufeisen, Britta / Neuner, Gerhard: Mehrsprachigkeitskonzept-Tertiärsprachen - Deutsch nach Englisch. Strassburg, Europarat. S. 13-34.

Ortner, Brigitte (2007): Motivierender Deutschunterricht: die Rolle der Lehrerinnen und Lehrer. In: Sammelband. VIII. Tagung des Verbandes der Deutschlehrer und Germanisten der Slowakei. Nitra, Spoločnosṫ učitelov nemeckého jazyka a germanistov Slovenska. S. 43-54.

Profil vykonávacích politických nástrojov jazykového vzdelávania. Slovenská republika. (2009) Divízia jazykovej politiky Rady Európy, Ministerstvo školstva Slovenskej republiky. Bratislava, Śtátny pedagogický ústav.

Raasch, Albert (2002): Europäische Sprachenpolitik. In: Zbornik prispevkov zo VI. konferencie Spoločnosti učitelov nemeckého jazyka a germanistov Slovenska. Bratislava, Spoločnost́ učitel'ov nemeckého jazyka a germanistov Slovenska. S. 28-32.

Riemer, Claudia (1997): Individuelle Unterschiede im Fremdsprachenerwerb. Die Wechselwirksamkeit ausgewählter Einflussfaktoren. Hohengehren, Schneider.

Rost-Roth, Sabine (2003): Förderung interkultureller Kompetenzen im Tertiärsprachenunterricht Deutsch nach Englisch. In: Hufeisen, Britta / Neuner, Gerhard (Hg.): Mehrsprachigkeitskonzept - Tertiärsprachen - Deutsch nach Englisch. Strassburg, Europarat. S. 51-84.

Schlak, Torsten / Banze, Kathrin / Haida, Justine / Kiline, Tefide / Kirchner, Katharina / Riemer, Claudia (2002): Die Motivation von DaF-Lernenden an Sprachlehrinstitutionen im Bielefelder Raum: Projektbeschreibung und erste Ergebnisse. H. 7(2). http://www.spz. tu-darmstadt.de/projekt_ejournal/jg_07_2/beitrag/schlak1.htm (30.6. 2004).

Steinig, Wolfgang / Huneke, Hans-Werner (2007): Sprachdidaktik Deutsch. Eine Einführung. Grundlagen der Germanistik - 38, 3. Aufl. Berlin, Erich Schmidt Verlag.

Tönshoff, Wolfgang (2000): Aktuelle Perspektiven für den fremdsprachlichen Deutschunterricht. In: Zbornik prispevkov z V. konferencie Spoločnosti učitelov nemeckého jazyka a germanistov Slovenska, 6.-9. septembra $2000 v$ Trnave. Trnava, Spoločnost́ učitelov nemeckého jazyka a germanistov Slovenska. S. 57-60.

Vaňková, Lenka (2007): Eröffnungsvortrag: Germanistik an tschechischen Universitäten: Gegenwart und Perspektiven. In: Ondráková, Jana / Vaňková, Lenka (Hg.): Germanistik an tschechischen Universitäten: Gegenwart und Zukunft. Referate der Konferenz des Tschechischen Germanistenverbandes, Hradec Králové, 12.-13. Oktober 2006. Universität Ostrava, Philosophische Fakultät. S. 11-15.

Wildenauer-Józsa, Doris (2004): „Ich vergleiche immer..."Wie erwachsene Deutschlernende ihre Sprachkenntnisse einsetzen: Beispiel Grammatik. In: Fremdsprache Deutsch. Zeitschrift für die Praxis des Deutschunterrichts 31/2004. S. 38-42. 